Chirurg 2006 $77: 111-116$ DOI 10.1007/s00104-006-1151-1

Online publiziert: 19. Januar 2006

(c) Springer Medizin Verlag 2006

M.-A. Ortner · G. Dorta

Department Gastroenterology and Hepatology, Centre Hospitalier Universitaire Vaudois, Lausanne, Schweiz

\title{
Endoskopische Diagnostik und Therapie der gastrointestinalen Blutung
}

\section{Diagnose der akuten Blutung}

In der Diagnosestellung hat sich bei der akuten Blutung gegenüber den letzten Jahren nicht viel geändert (• Abb. 1). An erster Stelle steht unverändert die klinische Erfassung der Schwere und vermutlichen Lokalisation der Blutung. Gastroskopie als Erstuntersuchung wird bei Hämatemesis und Meläna durchgeführt und die Koloskopie nach Kolonpräparation bei Hämatochezie.

Der ideale Zeitpunkt der Endoskopie wird kontrovers diskutiert. Zirka 70-80\% der GI-Blutungen kommen spontan zum Stillstand. Trotzdem galt bisher die Empfehlung, die Endoskopie sofort durchzuführen. Dies ist bei Verdacht auf eine schwere Varizenblutung weiterhin erforderlich. Neuere Arbeiten weisen darauf hin, dass bei den übrigen Blutungen eine elektive Endoskopie innerhalb der ersten $24 \mathrm{~h}$ ausreichend ist $[29,4]$. Die sofortige Endoskopie wurde mit der elektiven Endoskopie verglichen. Dabei zeigten sich keine Unterschiede bezüglich primärer Blutstillung, chirurgischer Intervention, Rezidivblutung, Komplikationsrate, Länge des Spitalsaufenthaltes und Mortalität. In beiden Arbeiten wurden bei der Gruppe mit sofortiger Intervention mehr spritzende Blutungen (Forrest Ia, $\bullet$ Tabelle 1) gefunden als bei der elektiven Endoskopie, obwohl die präendoskopische Risikoevaluierung (Rockall-Score, • Tabelle 2) identisch war $[9,27]$.

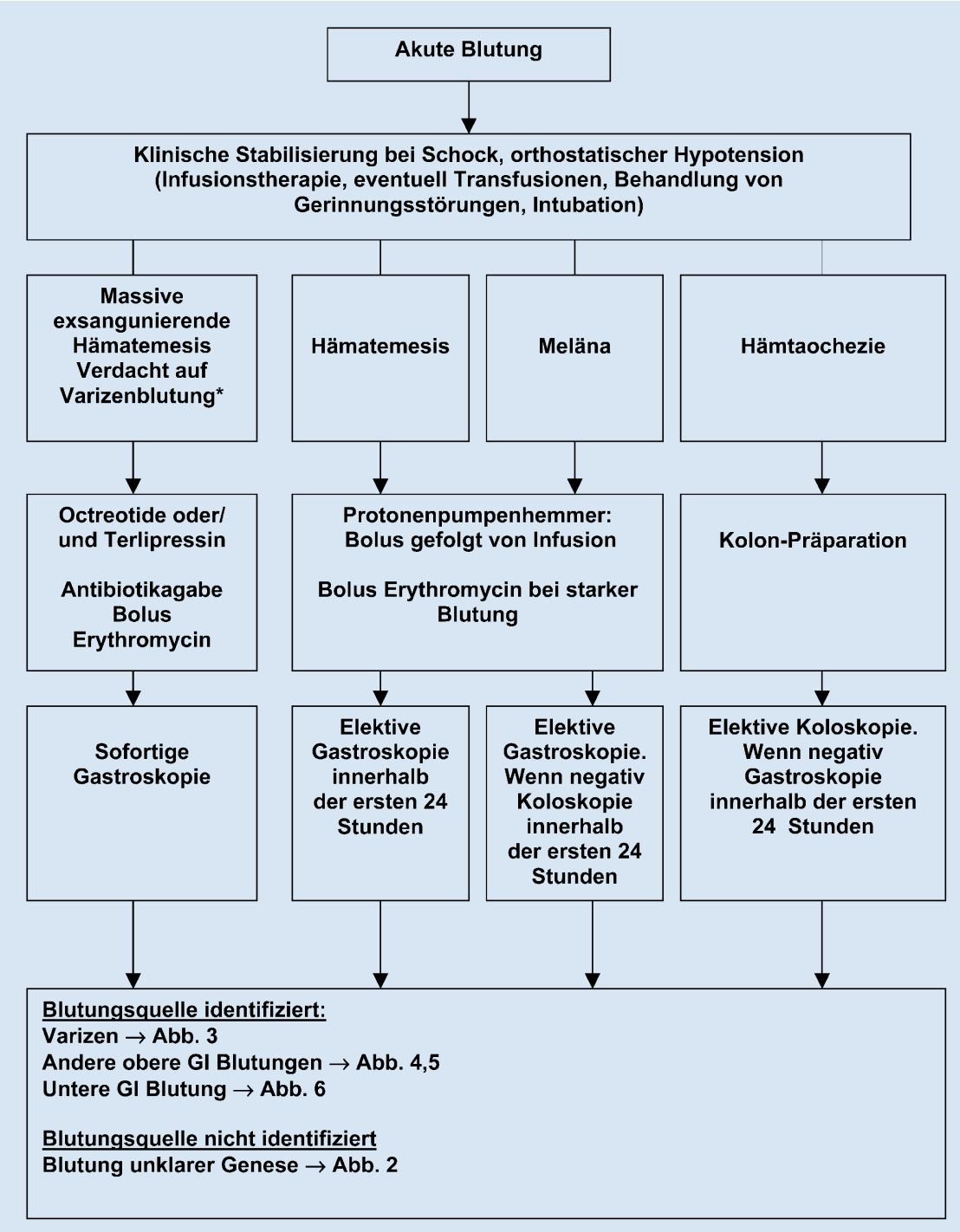

Abb. $1 \Delta$ Diagnose der akuten Blutung (*bekannte portale Hypertension, Varizen oder Zirrhose) 
- Daraus kann man folgern, dass einige Blutungen im Verlaufe der $24 \mathrm{~h}$ Wartezeit spontan, auch ohne endoskopische Therapie, zum Stillstand kommen.

Da der Verlauf durch zusätzliche medikamentöse Therapie verbessert wird, empfiehlt sich, den Patienten bereits im Rahmen der klinischen Stabilisierung anzubehandeln. Bei Verdacht auf Varizenblutung wird Octreotide (Bolus $50 \mu \mathrm{g}$ gefolgt von Infusion mit $50 \mu$ g über 5 Tage) und/oder Terlipressin (1-2 mg intravenös alle $4 \mathrm{~h}$ über 1-7 Tage) und bei Verdacht auf Blutungen anderer Genese ein Protonenpumpenhemmer (Bolus $80 \mathrm{mg}$ intravenös gefolgt von Infusion mit $8 \mathrm{mg} / \mathrm{h}$ ) verabreicht $[8,13,2]$.

Eine erfolgreiche endoskopische Therapie erfordert eine Identifizierung der Blutungsquelle. Die endoskopische Sicht ist vor allem bei schweren Blutungen häufig durch nichtentfernbare Blutkoagel ein-

geschränkt. Deshalb empfiehlt sich die Gabe von Erythromycin (Bolus $3 \mathrm{mg} / \mathrm{kg}$ Körpergewicht intravenös über $20 \mathrm{~min}$ ) ungefähr 30 min vor Endoskopie. Dadurch kann die Blutungsquelle besser identifiziert, die Hämostase verbessert und die Untersuchungszeit verringert werden [10].

Durch eine antibiotische Prophylaxe bei Ösophagusvarizenblutung können Infektionsrate, Rezidivblutungsrate und Transfusionsbedarf gesenkt werden [26]. Derzeit werden vor allem Quinolonpräparate empfohlen (z. B. Ciprofloxacin 400-1000 mg i. v. gefolgt von oralem Ciprofloxacin für 7-10 Tage).

\section{Blutung unklarer Genese}

Bei der Blutung unklarer Genese empfiehlt sich vor weiteren Maßnahmen eine Regastroskopie, durchgeführt von einem erfahrenen Kollegen, denn zahlreiche der

\section{Tabelle 1}

\section{Blutungsstigmata (Forrest-Kriterien)}

\section{Forrest-Kriterien}

Forrest I

- la

- Ib

Forrest II

- lla

- Ilb

- Ilc

Forrest III
Endoskopische Zeichen

Aktive Blutung

Spritzend

Sickernd

Zeichen einer stattgehabten Blutung

Sichtbarer Gefäßstumpf

Ulkus mit anhaftendem Blutkoagel

Ulkus mit Hämatinbelägen

Keine Zeichen einer stattgehabten Blutung

Ulkus mit unauffälligem Grund zunächst verpassten Läsionen sind mit dem Standardendoskop zugänglich [30] (- Abb. 2). Bei massiver, anhaltender Blutung ist die Angiographie mit der Möglichkeit einer gleichzeitigen Therapie der Szintigraphie vorzuziehen. Bei unklaren Blutungen können mit der Kapselendoskopie mehr Läsionen lokalisiert werden als mit der Enteroskopie [1]. Im Rahmen einer Blutungsepisode bietet die Enteroskopie jedoch den Vorteil der sofortigen Therapie (alternativ kann, wenn weder Ballon- noch Pushenteroskopie zur Verfügung stehen, ein pädiatrisches Koloskop verwendet werden).

\section{Therapie der Varizenblutung}

Die Ligaturbehandlung hat in den letzten Jahren die Sklerotherapie wegen ihres besseren Sicherheitsprofils und ihrer größeren Effektivität als Erstlinientherapie abgelöst. Rezidivprophylaxe mit Ligatur und einem nichtselektiven $\beta$-Blocker reduziert die Rezidivblutungsrate und die Mortalität [23] und ist die derzeit kostengünstigste $\mathrm{Me}$ thode [28] (siehe hierzu auch den Beitrag von Kölz u. Arn in diesem Heft). Ob in $\mathrm{Zu}$ kunft eine Ligatur-Sklerotherapie-LigaturSandwich-Technik [11], allenfalls nach Risikostratifizierung mittels endoskopischem Ultraschall, die Rezidivblutungsgefahr und damit die Mortalität senken kann, muss sich erst zeigen [14] (• Abb. 3).

Histoacryl $^{\circ}$ stellt weiterhin den Goldstandard in der Behandlung von FundusCardia- und Korpusvarizen dar [24] und

Tabelle 2

\section{Rockall-Score}

\begin{tabular}{|c|c|c|c|c|}
\hline & 0 & 1 & 2 & 3 \\
\hline Alter [Jahre] & $<60$ & $60-79$ & $\geq 80$ & \\
\hline \multirow[t]{3}{*}{ Schock } & Keiner & Tachykardie & Hypotension & \\
\hline & Puls $<100$ & $R R \geq 100$ & $\mathrm{RR}<100$ & \\
\hline & $R R \geq 100$ & Puls $\geq 100$ & & \\
\hline Komorbidität & Keine & & $\begin{array}{l}\text { Kardiale Insuffizienz und andere } \\
\text { schwere Erkrankungen }\end{array}$ & $\begin{array}{l}\text { Nierenversagen, Leberinsuffizienz, } \\
\text { disseminierte Krebserkrankung }\end{array}$ \\
\hline \multirow[t]{2}{*}{ Diagnose } & Mallory Weiss & Alle anderen & & \\
\hline & Keine Läsion & Diagnosen & & \\
\hline Endoskopische Stigmata & Keine oder Hämatin & & Koagel, aktive Blutung, & \\
\hline & & & Blut im oberen GI-Trakt & \\
\hline
\end{tabular}

RR systolischer Druck ( $\mathrm{mm} \mathrm{Hg}$ ). 


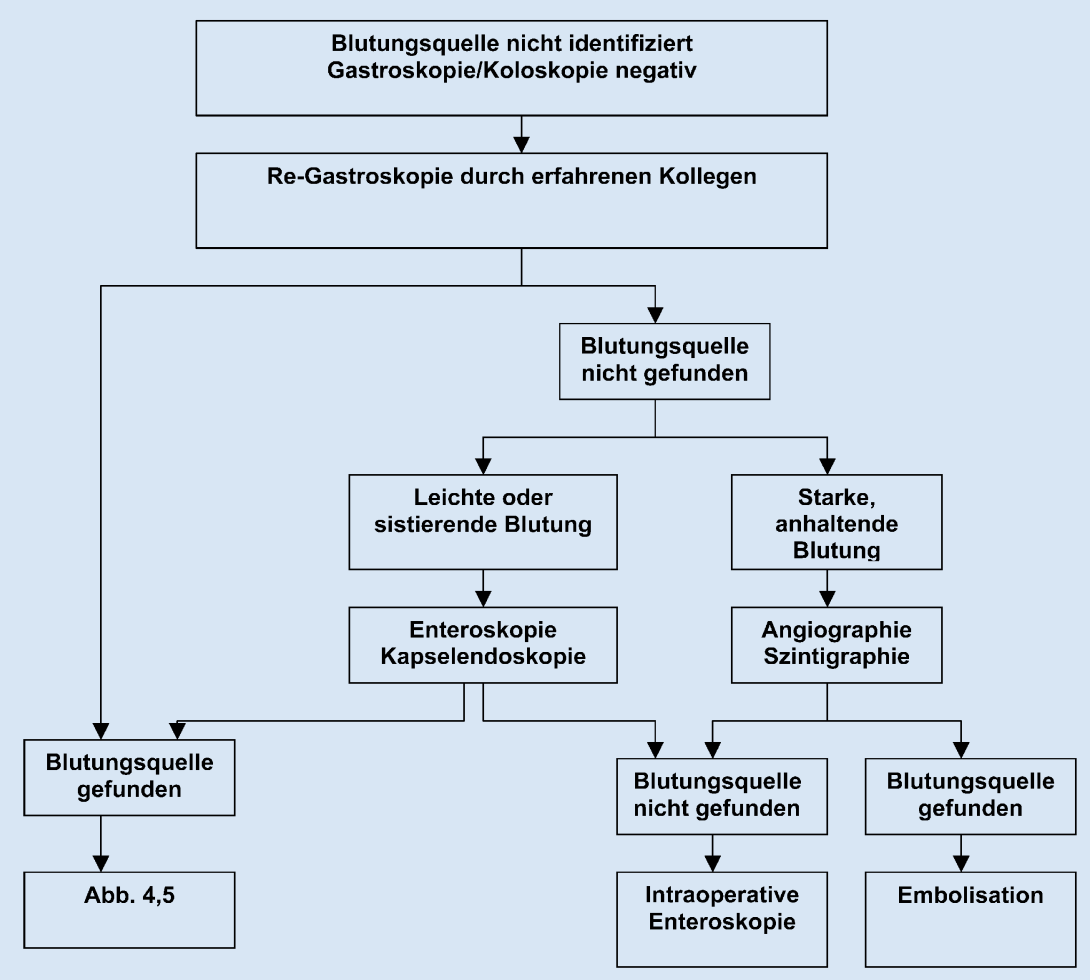

Abb. $2 \triangle$ Blutung unklarer Genese

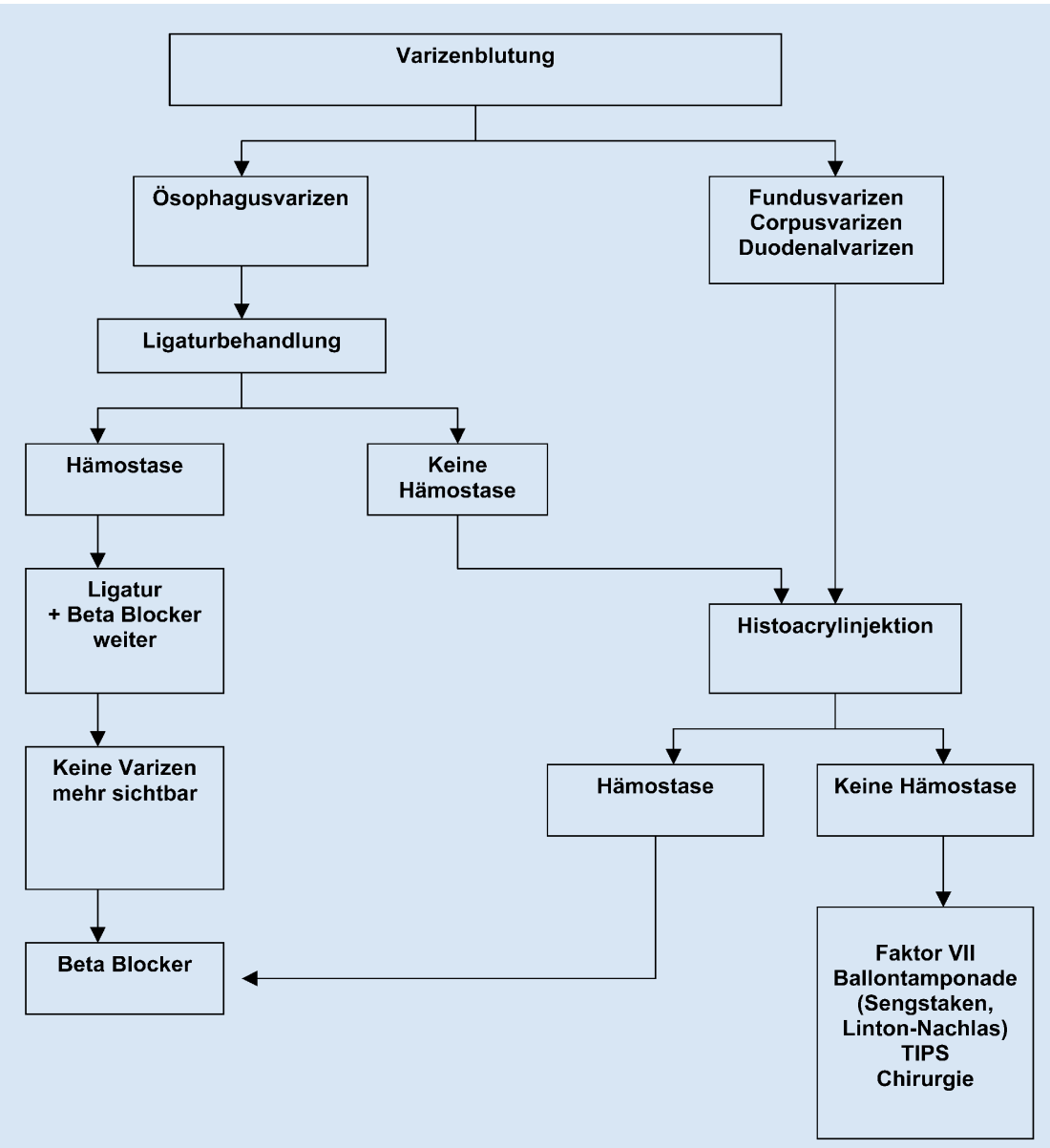

Abb. $3 \Delta$ Therapie der Varizenblutung
Chirurg 2006 $77: 111-116$

DOI 10.1007/s00104-006-1151-1

○) Springer Medizin Verlag 2006

\section{M.-A. Ortner - G. Dorta}

\section{Endoskopische Diagnostik und Therapie der gastrointestinalen Blutung}

\section{Zusammenfassung}

Die gastrointestinale Blutung stellt unveränderte einen der häufigsten medizinischen Notfälle dar. Obwohl die Mortalität in den letzten Jahren durch Verbesserung der endoskopischen Diagnostik und Therapie leicht gesenkt werden konnte, liegt sie immer noch bei $15 \%$. Zahlreiche Publikationen zu neuen Techniken und Therapiestrategien zeigen kontroverse Ergebnisse, so dass der Endoskopiker in seinen Therapienentscheidungen häufig auf seine persönliche Erfahrung angewiesen ist. Daher werden in diesem Artikel, neben einer neuerer Literaturanalyse zu diesem Thema, Therapieempfehlungen für die Praxis aus dem eigenen Erfahrungsbereich gegeben.

\section{Schlüsselwörter}

Akute Blutung · Blutung unklarer Genese . Varizenblutung · Ulkusblutung · Obere gastrointstinale Blutung · Untere gastrointstinale Blutung

\section{Endoscopic diagnosis and therapy for gastrointestinal bleeding}

\section{Abstract}

Gastrointestinal bleeding is still one of the most frequent medical emergencies. Despite improvements in endoscopic diagnosis and therapy, mortality from bleeding is still high (15\%). Since conclusive trials are lacking, the endoscopist often has to rely on personal experience in the selection of therapeutic options. Therefore this article gives an overview of new publications in this field and recommendations based on personal experience.

\section{Keywords}

Acute bleeding - Obscure bleeding . Variceal hemorrhage - Bleeding ulcers . Upper gastrointestinal bleeding $\cdot$ Lower gastrointestinal bleeding 


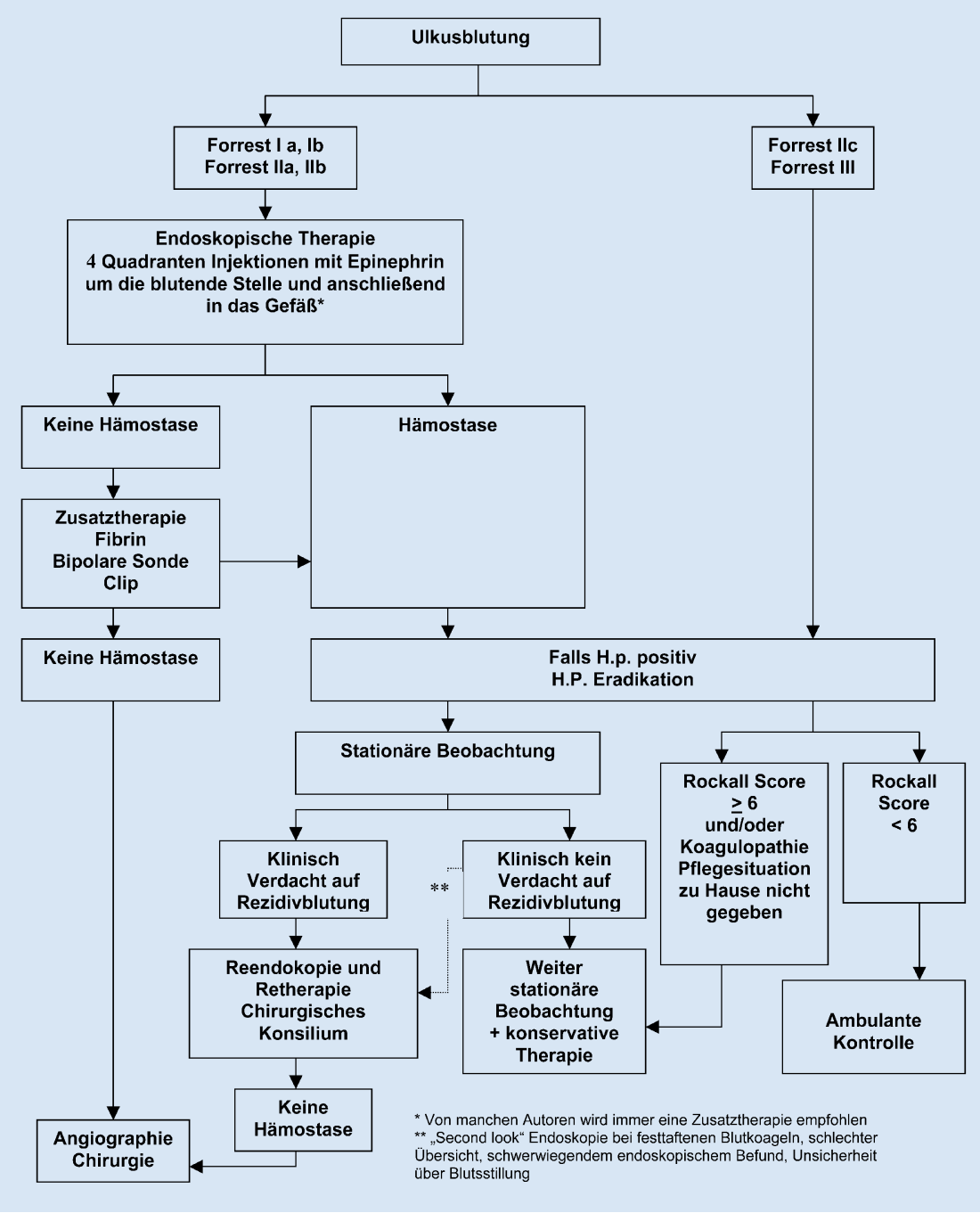

Abb. $4 \Delta$ Therapie der Ulkusblutung

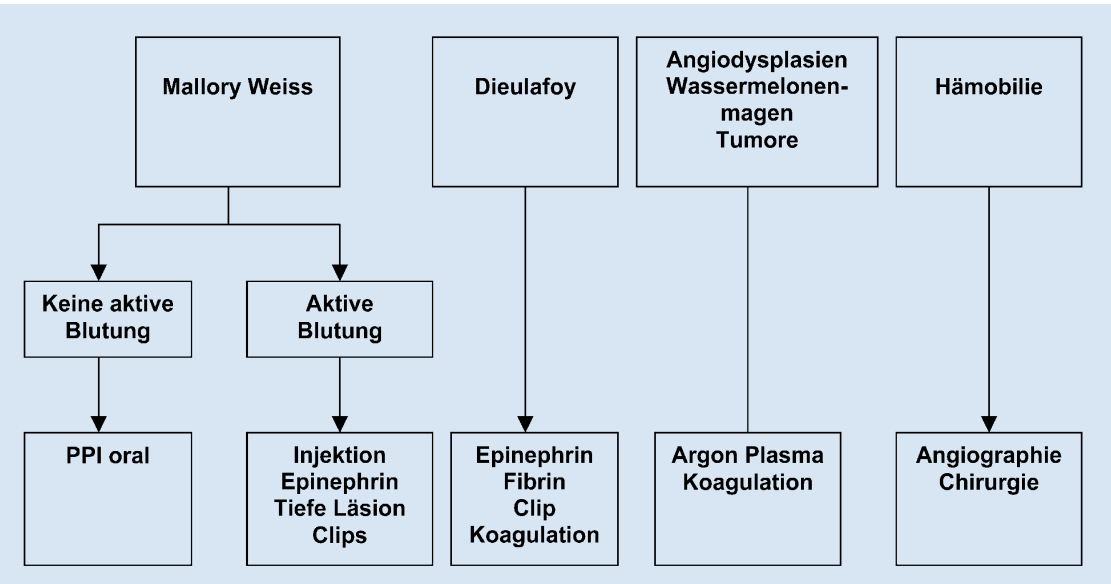

Abb. $5 \Delta$ Therapie der oberen GI-Blutung - seltene Ursachen kann bei Therapieversagen der Ligaturbehandlung auch bei den Ösophagusvarizen mit Erfolg eingesetzt werden.

\section{- Die Ballontamponade (Sengstaken- Blakemore- oder Linton-Nachlas-Son- de) sollte wegen des hohes Rezidivblu- tungsrisikos und der Perforationsge- fahr nur bei Therapieversagern ange- wandt werden.}

Eventuell könnte die Sondeneinlage in $\mathrm{Zu}$ kunft durch die Gabe des aktivierten rekombinanten Faktors VII (8o $\mu \mathrm{g} / \mathrm{kg}$ Körpergewicht) abgelöst werden [5].

\section{Therapie der Ulkusblutung}

Die Rezidivblutung ist der wichtigste prognostische Faktor (• Abb. 4). Sie ist mit einem 15fachem Anstieg der Mortalität assoziiert. Zur Therapieplanung werden Rockall-Score (• Tabelle 2) und die Forrest-Kriterien (• Tabelle 1) herangezogen (siehe hierzu auch den Beitrag von Knoefel u. Rehders in diesem Heft). Beim Rockall-Score liegt der Trennwert zwischen Hochrisikopatienten und Patienten mit geringem Risiko bei 6 [3]. Bei den Forrest-Stadien sind vor allem die Stadien I und IIa mit einem erhöhten Risiko assoziiert, und es ist hier eine klare Indikation zur endoskopischen Therapie gegeben.

Trotz kontroverser Debatten bezüglich der Forrest-Ilb-Ulzera bevorzugen wir auch hier eine endoskopische Therapie, da die Rezidivblutungsrate dadurch gesenkt werden kann [17].

Forrest-Stadien IIc und III werden nur medikamentös behandelt (Protonenpumpenhemmer, Helicobacter-pylori-Eradikation bei positivem Befund) [19]. Bei niederem Rockall-Score, normaler Gerinnung und gegebener Pflegesituation können diese Ulzera ambulant weiter betreut werden $[3,6]$.

Die Injektionstherapie von Epinephrin ist unverändert die Methode erster Wahl (1:10 00o) [20]. Mit Injektion größerer Volumina $(13-20 \mathrm{ml})$ verbessert sich sowohl die initiale Blutstillungsrate als auch das Rezidivblutungsrisiko [22]. EineZusatzme- 
thode (Fibrininjektion, elektrische Koagulation, Clip) zur definitiven Blutstillung ist bei großen Gefäßen oder aktiver Blutung manchmal notwendig. Von anderen Autoren wird die elektrische oder thermische Koagulation oder die Kombination Injektion/Elektrokoagulation als Erstlinientherapie bevorzugt. Ein endoskopisches Dopplersignal ist nicht hilfreich, um das Rezidivblutungsrisiko und damit die Therapiestrategie zu bestimmen [15].

Bei Rezidivblutung verbessert eine erneute endoskopische Therapie den Verlauf bei Hochrisikopatienten [7]. Eine routinemäßige „Second-look-Endoskopie“ verbessert den Verlauf jedoch nicht [25].

\section{Therapie seltener Ursachen der oberen GI-Blutung}

Die meisten Mallory-Weiss-Blutung stoppen spontan. Bei aktiver Blutung (Koagulationsstörung, hämodynamische Instabilität) kann eine Therapie mit Injektion [21] oder Clip [12] unternommen werden (- Abb. 5). Der Clip sollte vor allem bei tieferen Läsionen verwendet werden, da hier bei Injektion die Gefahr einer Perforation gegeben ist. Dieulafoy-Läsionen werden wie Ulzera mittels Injektion, Clip oder Koagulation behandelt [31].

Für Angiodysplasien, antrale vaskuläre Ektasien oder Tumore eignet sich die Therapie mit Argon-Plasma-Koagulation unverändert am besten [18]. Bei Hämobilie empfiehlt sich immer eine Angiographie, um ein Aneurysma, Pseudoaneurysma oder eine arterioportale-venöse Fistel auszuschließen.

\section{Therapie der unteren GI-Blutung}

In diesem Bereich gibt es kaum neue Studien [16] und am diagnostischen und therapeutischen Vorgehen hat sich im Laufe der Jahre nichts verändert (• Abb. 6; siehe hierzu auch den Beitrag von Klar et al. in diesem Heft).

\section{Korrespondierender Autor Dr. M.-A. Ortner}

Department Gastroenterology and Hepatology, Centre Hospitalier Universitaire Vaudois, Rue du Bugnon 46, 1011 Lausanne, Schweiz E-Mail: Maria-Anna.Ortner@chuv.ch
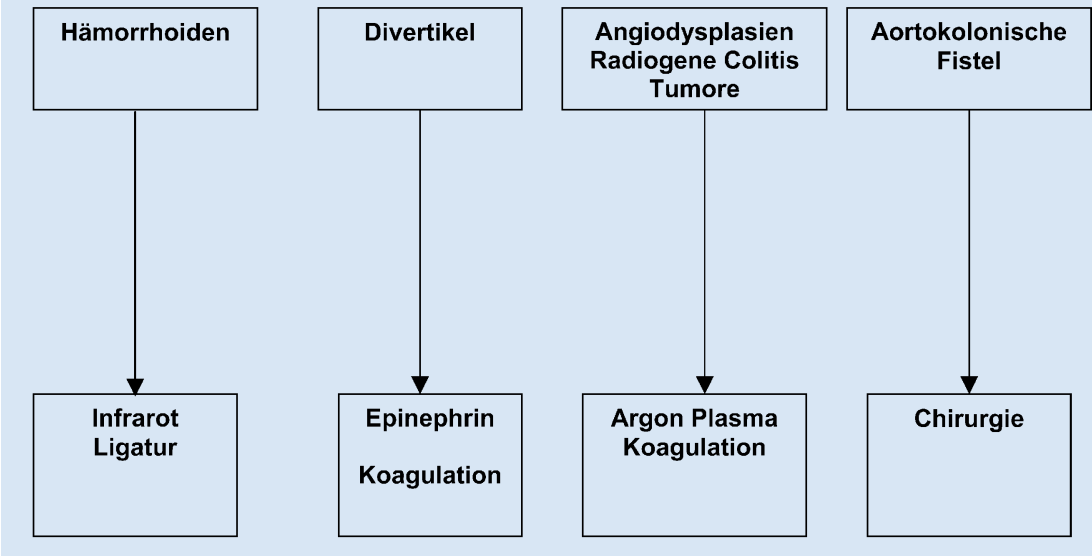

\section{Abb. $6 \Delta$ Therapie der unteren GI-Blutung}

Interessenkonflikt: Es besteht kein Interessenkonflikt. Der korrespondierende Autor versichert, dass keine Verbindungen mit einer Firma, deren Produkt in dem Artikel genannt ist, oder einer Firma, die ein Konkurrenzprodukt vertreibt, bestehen. Die Präsentation des Themas ist unabhängig und die Darstellung der Inhalte produktneutral.

\section{Literatur}

1. Adler DG, Knipschield M, Gostout C (2004) A prospective comparison of capsule endoscopy and push enteroscopy in patients with GI bleeding of obscure origin. Gastrointest Endosc 59:187-188

2. Bardou M, Toubouti Y, Benhaberou-Brun D, Rahme E, Barkun AN (2005) Meta-analysis: proton-pump inhibition in high-risk patients with acute peptic ulcer bleeding. Aliment Pharamcol Ther 21:677-686

3. Bessa X, Callaghan EO, Ballesté B et al. (2006) Applicability of the Rockall score in patients undergoing endoscopic therapy for upper gastrointestinal bleeding. Dig Dis 25 (in press)

4. Bjorkman DJ, Zaman $A$, Fennerty MB, Lieberman S, Disario JA, Guest-Warnick G (2004) Urgent vs. elective endoscopy for acute non-variceal upper $\mathrm{Gl}$ bleeding: an effectiveness study. Gastrointest Endosc 60:94-95

5. Bosch J, Thabut D, Bendtsen F et al. European Study Group on rFVIla in UGI haemorrhage (2004) Recombinant factor VII a for upper gastrointestinal bleeding in patients with cirrhosis: a randomized, double-blind trial. Gastroenterology 127:11231130

6. Brullet E, Campo R, Calvet X, Guell M, Garcia-Monforte N, Cabrol J (2004) A randomized study of the safety of outpatient care for patients with bleeding peptic ulcer treated by endoscopic injection. Gastrointest Endosc 60:15-21

7. Calvet $X$, Vergara M, Brullet E, Gisbert JP, Campo R (2004) Addition of a second endoscopic treatment following epinephrine injection improves outcome in high-risk bleeding ulcers. Gastroenterology 126:441-450

8. D’Amico G, Piertosi G, Tarantino I, Pagliaro L (2003) Emergency sclerotherapy versus vasoactive drugs for variceal bleeding in cirrhosis: a Cochrane metaanalysis. Gastroenterology 124:1277-1291

9. Forrest JA, Finlayson ND, Shearman DJ (1974) Endoscopy in gastrointestinal bleeding. Lancet ii: 394-397
10. Frossard JL, Spahr L, Queneau PE et al. (2002) Erythromycin intravenous bolus infusion in acute upper gastrointestinal bleeding: a randomized, controlled, double-blind trial. Gastroenterology 123:17-23

11. Hou MC, Chen WC, Lin HC, Lee FY, Chang FY, Lee SD (2001) A new „sandwich" method of combined endoscopic variceal ligation and sclerotherapy versus ligation alone in the treatment of esophageal variceal bleeding: a randomized trial. Gastrointest Endoscopy 53:572-578

12. Huang SP, Wang HP, Lee YC et al (2002) Endoscopic hemoclip placement and epinephrine injection for Mallory Weiss syndrome with active bleeding. Gastrointest Endoscopy 55:842-846

13. loannou GN, Doust J, Rockey DC (2003) Systemic review: terlipressin in acute esophageal variceal hemorrhage. Aliment Pharmacol Ther 17:53-64

14. Irisawa A, Saitpo A, Obara K et al. (2001) Endoscopic recurrence of esophageal varices is associated with the specific EUS abnormalities: severe periesophageal collateral veins and large perforating veins. Gastrointest Endoscopy 53:77-84

15. Jakobs R, Zoepf T, Schilling D, Siegel EG, Riemann JF (2004) Endoscopic Doppler ultrasound after injection therapy for peptic ulcer hemorrhage. Hepatogastroenterology 51:1206-1209

16. Jensen DM, Machicado GA, Jutabha R, Kovacs $T O$ (2000) Urgent colonoscopy for the diagnosis and treatment of severe diverticular hemorrhage. $\mathrm{N}$ Engl J Med 342:78-82

17. Kahi CJ, Jensen DM, Sung JJ, Bleau BL, Jung HK, Eckert G, Imperiale TF (2005) Endoscopic therapy versus medical therapy for bleeding peptic ulcer with adherent clot: a meta-analysis. Gastroenterology 129:855-862

18. Kanai M, Hamada A, Endo Y, Takeda Y, Yamakawa M, Nishikawa H, Torii A (2004) Efficacy of argon plasma coagulation in nonvariceal upper gastrointestinal bleeding. Endoscopy 36:1085-1088

19. Kikkawa A, Iwakiri R, Ootani H et al. (2005) Prevention of the rehaemorrhage of bleeding peptic ulcers: effects of helicobacter pylori eradication and acid suppression. Aliment Pharmacol Ther 2:79-84

20. Laine L, Estrada R (2002) Randomized trial of normal saline solution injection versus bipolar electrocoagulation for treatment of patients with highrisk bleeding ulcers: is local tamponade enough? Gastrointest Endoscopy 55:6-10

21. Liach J, Elizalde Ji, Guevera MC et al. (2001) Endos copic injection therapy in bleeding Mallory-Weiss syndrome: a randomized controlled trial. Gastrointest Endosc 54:679-681 
22. Lin HJ, Hsieh HY, Tseng GY et al. (2002) A prospective randomized trial of large-versus small volume endoscopic injection of epinephrine for peptic ulcer bleeding. Gastrointest Endoscopy 55:615-619

23. Lo GH, Lai KH, Cheng JS, Chen MH, Huang JS, Hsu $\mathrm{Pl}$, Lin CK (2000) Endoscopic variceal ligation plus nadolol and sucralfate compared with ligation alone for the prevention of variceal rebleeding: a prospective, randomized trial. Heaptology 32:461465

24. Lo GH, Lai KH, Cheng JS, Chen MH, Chiang HT (2001) A prospective, randomized trial of butyl cyanoacrylate injection versus band ligation in the management of bleeding gastric varices. Hepatology 33:1060-1064

25. Messmann H, Schaller P, Andus T et al. (1998) Effect of programmed endoscopic follow-up examination on the rebleeding rate of gastric or duodenal peptic ulcers treated by injection therapy: a prospective, randomized controlled trial. Endoscopy 30:650-652

26. Pohl J, Pollmann K, Sauer P, Ring A, Stremmel W, Schlenker T (2004) Antibiotic prophylaxis after varíceal hemorrhage reduced incidence of early rebleeding. Hepatogastroenterology 51:541-546

27. Rockall Ta, Logan RFA, Devlin HB, Northfield TC (1996) Risk assessment after acute upper gastrointestinal hemorrhage. Gut 38:316-321

28. RubensteinJH, Eisen GM, Inadomi JM (2004) A cost-utility analysis of secondary prophylaxis for variceal hemorrhage. Am J Gastroenterol 99:12741288

29. Schacher GM, Pantoflickova DL, Ortner MA; Wasserfallen JB, Blum AL, Dorta G (2004) Is early endoscopy in the emergency room beneficial in patients with bleeding peptic ulcer? A ,fortuitously controlled" study. Endoscopy 37:1-5

30. Triester SL, Leighton JA, Leontiadis Gl et al. (2005) OA meta-analysis of the yield of capsule endoscopy compared to other diagnostic modalities in patients with obscure gastrointestinal bleeding. Am J Gastroenterol 100:2407-2418

31. Yoshida T, Adachi K, Tanioka Y et al. (2004) Dieulafoy's lesion of the esophagus correctly diagnosed and successfully treated by the endoscopic injection of N-butyl-2cyanoacrylate. Endoscopy 36:183185

\section{Röntgen Fotografie Wettbewerb 2006}

Das Medizin zugleich auch eine Kunst ist, darüber ist man sich in Fachkreisen einig. Der Röntgen Fotografie Wettbewerb 2006 - der Wettbewerb für kreative Ärzte - richtet sich an alle Mediziner, die sich als Röntgenkünstler abseits des Praxisalltags versuchen möchten. Teilnehmen kann jeder, der eine ausgefallene Idee zum Thema Röntgenfotografie beizusteuern hat. Prämiert werden die Kunstwerke von einer Fachjury aus Journalisten, Fotografen und Gestaltern

Die originellsten Ideen werden mit Preisen im Gesamtwert von über 5000 Euro prämiert. Die Röntgenfotografien in digitaler oder analoger Form können bis zum 31. März 2006 an unten stehende Adresse eingesendet werden. Mehr Details zur Anmeldung, Abgabe und Bewertung sind auf der Wettbewerbshomepage www. roentgenfotografie.de zu finden.

Wettbewerbsanschrift:

Arzt\&Praxis GmbH

Gesellschaft für EDV-gestützte Systeme

in der Medizin $\mathrm{GmbH}$

Stichwort »Röntgenfotografie»

Maybachstraße 39

70469 Stuttgart

E-Mail:info@roentgenfotografie.de

\section{OPS 2006}

Endgültige Fassung beim DIMDI veröffentlicht

Das Deutsche Institut für Medizinische Dokumentation und Information (DIMDI) hat die endgültige Fassung des Operationenund Prozedurenschlüssels (OPS), Version 2006, auf seinen Internetseiten veröffentlicht. Viele Prozeduren können mit der neuen Version präziser und differenzierter verschlüsselt werden als bisher.

Der OPS 2006 ist eine der Grundlagen für das pauschalierende Vergütungssystem in Klinik und Praxis (German Diagnosis Related Groups, G-DRG-System).
Wesentliche Änderungen im Systematischen Verzeichnis des OPS 2006 sind

- die Umstellung auf die neue Recht schreibung,

- umfangreiche Überarbeitung der HNOOperationen,

- Überarbeitung und Ergänzung der Codes in den Bereichen Frührehabilita tion und Komplexbehandlung,

- neue Codes für zusatzentgeltpflichtige Medikamente.

Das Alphabetische Verzeichnis zum OPS 2006 wurde deutlich erweitert. Neben zahlreichen Ergänzungen und Verbesserungen wurden alle Änderungen des Systematischen Verzeichnisses in das Alphabetische Verzeichnis eingearbeitet. Es enthält jetzt mehr als 60.000 Sucheinträge.

Der Kommentar im Vorspann des Systematischen Verzeichnisses gibt einen schnellen Überblick über wichtige Neuerungen. Alle Änderungen gegenüber der OPS-Version 2005 sind im Detail in einer Aktualisierungsliste auf den Internetseiten des DIMDI zusammengefasst.

Informationen über das Herunterladen der Referenzausgaben des OPS 2006 sowie über das Bestellen von Buchausgaben des OPS 2006 sind auf der Webseite des DIMDI (www.dimdi.de) zu finden.

Quelle: Arbeitsgemeinschaft der Wissenschaftlichen Medizinischen Fachgesellschaften (AWMF), www.uni-duesseldorf. de/WWW/AWMF/;

Deutsches Institut für Medizinische Dokumentation und Information (DIMDI), www.dimdi.de 